\title{
DEVELOPMENT OF MOTORIZED SLEWING MIRROR STAGE FOR THE UFFO PROJECT
}

J. Nam ${ }^{1}$, for the UFFO Collaboration, K.B. $\mathrm{Ahn}^{2}, \mathrm{M} \mathrm{Cho}^{3}$, S. Jeong ${ }^{3}$, J.E. Kim ${ }^{3}$, S. Ahmad ${ }^{4}$, P. Barrillon ${ }^{4}$, S. Brandt ${ }^{5}$, C. Budtz-Jørgensen ${ }^{5}$,

A.J. Castro-Tirado ${ }^{6}$, C.-H. Chang $^{7}$, C.-Y. Chang ${ }^{7}$, Y.Y. Chang ${ }^{1}$, C.R. Chen ${ }^{7}$, P. Chen ${ }^{1}$, H.S. Choi ${ }^{8}$, Y.J. Choi ${ }^{9}$, P. Connel ${ }^{10}$, S. Dagoret-Campagne ${ }^{4}$, C. Eyles ${ }^{10}$, B. Grossan ${ }^{3}$, J.J. Huang ${ }^{1}$, M.-H.A. Huang ${ }^{11}$, A. Jung ${ }^{3}$, M.B. Kim ${ }^{12}$, S.-W. Kim ${ }^{2}$, Y.W. Kim ${ }^{12}$, A.S. Krasnov ${ }^{13}$, J. Lee ${ }^{12}$, H. Lim ${ }^{12}$, E.V. Linder ${ }^{3}$, T.-C. Liu ${ }^{1}$, N. Lund ${ }^{5}$, K.W. $\mathrm{Min}^{8}$, G.W. $\mathrm{Na}^{3}$, M.I. Panasyuk ${ }^{13}$, I.H. Park ${ }^{12}$, V. Reglero ${ }^{10}$, J. Ripa ${ }^{12}$, J.M. Rodrigo ${ }^{6}$, G.F. Smoot ${ }^{3}$, J.E. Suh ${ }^{3}$, S. Svertilov ${ }^{13}$, N. Vedenkin ${ }^{13}$, M.-Z. Wang ${ }^{1}$ and I. Yashin ${ }^{13}$

\begin{abstract}
The Ultra-Fast Flash Observatory (UFFO) is a space observatory for optical follow-ups of gamma ray bursts (GRBs), aiming to explore the first 60 seconds of GRBs optical emission. UFFO is utilized to catch early optical emissions from GRBs within few sec after trigger using a Gimbal mirror which redirects the optical path rather than slewing entire spacecraft. We have developed a $15 \mathrm{~cm}$ two-axis Gimbal mirror stage for the UFFO-Pathfinder which is going to be on board the Lomonosov satellite which is to be launched in 2013 . The stage is designed for fast and accurate motion with given budgets
\end{abstract}

\footnotetext{
1 National Taiwan University, Taipei, Taiwan

2 Yonsei University, Seoul, Korea

3 Ewha Womans University, Seoul, Korea

4 LAL, University of Paris-Sud 11, Orsay, France

5 Technical University of Denmark, Copenhagen, Denmark

6 Instituto de Astrofísica de Andalucía, CSIC, Granada, Spain

7 National Space Organization, Hsinchu, Taiwan

8 Korea Institute of Industrial Technology, Cheonan, Korea

9 Korea Advanced Institute of Science and Technology, Deajeon, Korea

10 University of Valencia, Valencia, Spain

11 National United University, Miao-Li, Taiwan

12 Sungkyunkwan University, Suwon, Korea

13 Moscow State University, Moscow, Russia
} 
of $3 \mathrm{~kg}$ of mass and 3 Watt of power. By employing stepping motors, the slewing mirror can rotate faster than $15 \mathrm{deg} / \mathrm{sec}$ so that objects in the UFFO coverage $(60 \mathrm{deg} \times 60 \mathrm{deg})$ can be targeted in $\sim 1 \mathrm{sec}$. The obtained targeting resolution is better 2 arcmin using a close-loop control with high precision rotary encoder. In this presentation, we will discuss details of design, manufacturing, space qualification tests, as well as performance tests.

\section{Introduction}

Having a fast response of GRB telescope has been scientists' great dream from BeppoSAX (Boella et al. 1997) through SWIFT (Gehrels et al. 2004). Now, the dream has been realized by UFFO (Park et al. 2013; Chen et al. 2011) with a fast localization- and slewing-capability. For the first step, we have developed a pathfinder payload (UFFO-Pathfinder) with a mass of $\sim 20 \mathrm{~kg}$ for launch in 2013, while a full scale mission with $100 \mathrm{~kg}$ mass, UFFO-100, is being planned for future.

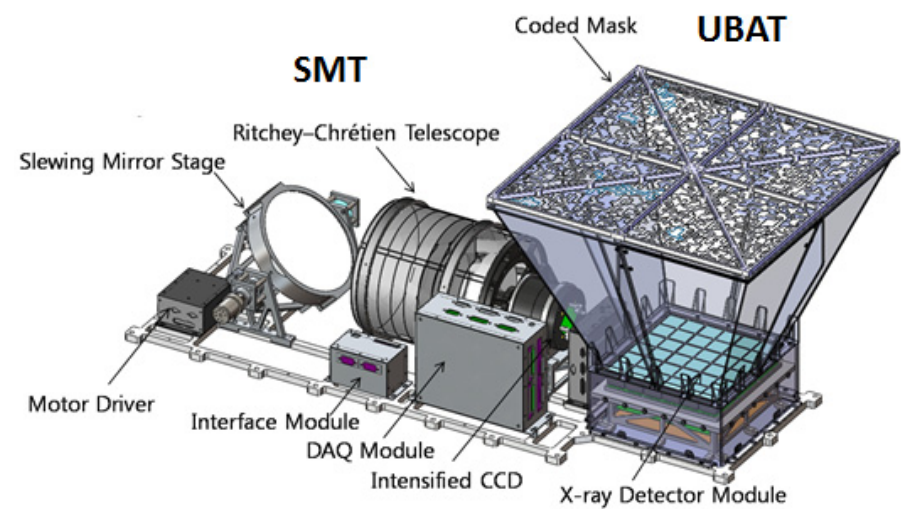

Fig. 1. A schematic view of UFFO-Pathfinder.

UFFO consists of two telescopes; the UFFO Burst Alert Telescope (UBAT) and the Slewing Mirror Telescope (SMT). UBAT is a $90^{\circ} \times 90^{\circ}$ wide Field Of View (FOV) coded mask X-ray camera to issue event triggers and to determine localizations. The localization can be obtained within a sec after the trigger using a fast imaging algorithm implemented in Field Programmable Gate Array (FPGA). The localization resolution is better than 10 arcmin in an energy range of X-rays 10-100 keV. More detailed information can be found in other references (Park et al. 2013; Jung et al. 2011).

SMT is the key concept of UFFO to capture early optical emissions from GRBs in the sub-minute regime (Jeong et al. 2013). SMT consists of three main instruments; Motorized Gimbal Mirror, Ritchey-Chrétien Telescope (RCT), and Intensified Charge-Coupled Device (ICCD) as a photo detector. The motorized 
Gimbal mirror is located in front of $\mathrm{RCT}$ to redirect the beam path. The $\mathrm{D}=10 \mathrm{~cm}$ RCT has a narrow FOV $(17$ arcmin $\times 17$ arcmin $)$, but the slewing capability further extends the SMT sky coverage up to 70 arcdeg $\times 70$ arcdeg. The pixel size of ICCD is $4 \operatorname{arcsec} \times 4$ arcsec. The Point Spread Function (PSF) of SMT is $\sim 1$ arcsec.

\section{Motorized Gimbal mirror system}

Figure 2 shows the motorized Gimbal mirror. In order to reduce the size, we designed a motor and the structure of the inner axis to be located below the mirror. $\mathrm{A} D=15 \mathrm{~cm}$ Zerodur substrate of the mirror was light-weighted to be $480 \mathrm{~g}$ with a light-weighting factor $\sim 60 \%$. In order to minimize mechanical stress, Silicone pads were inserted at six points round the mirror in the gap between the mirror and the mount ring. It also helps reduce deformation of the mirror surface by nonuniform thermal expansion. The enhanced-aluminum coating on mirror surface provides $\sim 85 \%$ of average reflectivity in a range of $200-700 \mathrm{~nm}$ wave length.

We used the unipolar type of stepper motors with a step size of $1.8 \mathrm{deg}$. Sufficient torque, as well as a fine size of step were obtained by employing a 100:1 gear on the output axis of the motor. To reduce steering uncertainty, Harmonic Drive Gears with a small backlash (less than 2 arcmin) were used. The minimum step size was further reduced to 4 arcsec using the 1/16 micro-stepping control technique. We used the close-loop control with a high precision rotary encoder placed on the mirror rotation axis at the opposite side from which the motor is located. The obtained targeting resolution was $\sim 2$ arcmin, limited by the accuracy of the rotary encoder. Maximum rotational speed of the mirror is faster than $15 \mathrm{deg} / \mathrm{sec}$ using a practical set of acceleration table. This corresponds to the optical targeting speed of $30 \mathrm{deg} / \mathrm{sec}$.

The motor control logic was implemented in FPGA. In order to reduce the power consumption, a single FPGA chip integrated all functions for both ICCD readout and motor control logic (Kim et al. 2011). While waiting for the trigger, the mirror stays at the standby mode targeting the center of UFFO FOV, where the average slewing angle for upcoming GRB events is expected to be the minimum. When a GRB event trigger occurs, the logic sends the motor drivers the number of pulses according to the angle from the standby position to the target coordinates.

SMT also receives triggers from the other X-ray instrument on the Lomonosov satellite; the BDRG. However, the success probability in targeting to BDRG events is expected to be low due to its limited localization resolution. The motor control includes compensation logic for the drift of the spacecraft (SC) based on interpolation of SC altitude information issued every second by the satellite. When the payload is exposed in the sunlight, the system parks the mirror in the safety mode where the mirror faces toward the zenith direction to minimize reflection of the sunlight on the mirror to optical instruments. Maximum power consumption of the mirror system is about $3.5 \mathrm{~W}$ including motors, drivers, and encoders. This power can be reduced to under $2 \mathrm{~W}$ by releasing the holding torque of the 


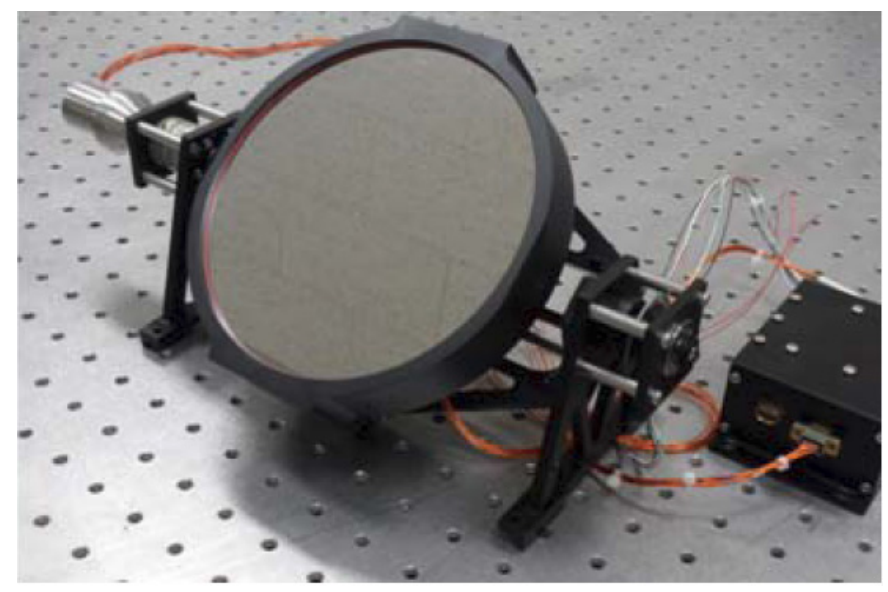

Fig. 2. Manufactured Motorized Gimbal Mirror System.

motors during the safety mode. Possible systematic errors such as optical axis mis-alignment and offsets of the rotary encoder can be corrected by parameters from calibrations during the flight.

\section{Test results and readiness}

The space qualification tests were performed with the fully integrated SMT system at the National Space Organization, Taiwan in Jul 2011. All devices including the motors, the encoders, the motor driver board, and the control board were found to be healthy under thermal vacuum conditions of the temperature range $-30 \sim 40^{\circ} \mathrm{C}$ and the pressure of $\sim 10^{-7}$ mbar. No outgassing material was found to contaminate the optical instruments. Subsequent shock $45 \mathrm{~g}$ for $3 \mathrm{~ms}$ ) and vibration (1$9.5 \mathrm{~g}$ in $5-2000 \mathrm{~Hz}$ ) tests were also successfully carried out without showing any instrumental damages or functional degradations. The UFFO has been integrated together with other payloads onto Lomonosov at Istra, Russia in 2012. The full system validation tests during the integration demonstrated successful targeting and imaging taking capabilities. Further developments on the mirror control are still in progress to optimize the slewing speed and settling time.

\section{Summary}

We have successfully developed the slewing mirror system for the UFFO-pathfinder. The UFFO-pathfinder will demonstrate the new concept for fast response telescope design by employing a slewing mirror on a space-based instrument, which will open up the promising future of scientific discoveries from GRBs during the very early photon emission phase. 


\section{References}

Boella, G., Butler, R.C., Perola, G.C., et al., 1997, A\&AS, 122, 299

Chen, P., Ahmad, S., Ahn, K.B., et al., 2011, Proceeding of ICRC [arXiv:1106.3929]

Gehrels, N., Chincarini, G., Giommi, P., et al., 2004, ApJ, 611, 1005

Jeong, S., Nam, J.W., Ahn, K.B., et al., 2013, Opt. express, 21, 2263

Jung, A., Ahmad, S., Ahn, K.B., et al., 2011, Proceeding of ICRC [arXiv:1106.3802]

Kim, J.E., Lim, H., Jung A., et al., 2011, Proceeding of ICRC [arXiv:1106.3803]

Park, I., Ahmad, S., Parrillon, P., et al., 2013, New J. Phys., 15, 023031 